\title{
P634A4 and TAp73 immunocytochemistry in liquid-based cervical cytology-potential biomarkers for diagnosis and progress prediction of cervical neoplasia
}

\author{
Annie NY Cheung ${ }^{1}$, Ka-Lai Tsun ${ }^{1}$, Kin-Man $\mathrm{Ng}^{1}$, Elaine Szeto ${ }^{1}$, Michelle K Siu ${ }^{1}$, \\ Esther S Wong ${ }^{1}$ and Hextan Y Ngan ${ }^{2}$ \\ ${ }^{1}$ Department of Pathology, The University of Hong Kong, Queen Mary Hospital, Hong Kong, China and \\ ${ }^{2}$ Department of Obstetrics and Gynecology, The University of Hong Kong, Queen Mary Hospital, Hong Kong, China
}

\begin{abstract}
$P 63$ and $p 73$ are two homologues of the important tumor suppressor gene $p 53$. In this study, we investigated p63 and p73 expression by immunocytochemistry using antibodies for TAp73 and p634A4 isoforms in 91 highgrade and 107 low-grade squamous intraepithelial lesions, 212 atypical squamous cells of undetermined significance, 9 squamous cell carcinomas and 63 normal samples from an Asian screening population together with 47 hospital samples of carcinomas. There was significant correlation between the TAp73 and p634A4 indices $(P<0.0001)$. Significantly, higher TAp73 and p634A4 indices were found in high-grade lesions or carcinoma when compared with atypical squamous cells and low-grade lesions $(P<0.0001)$. Among atypical squamous cells, p634A4 indices of cases that subsequently progressed to low-grade $(P=0.031)$ or high-grade lesions $(P=0.006)$ were significantly higher than those that did not. For atypical squamous cells positive for high-risk human papillomavirus (HPV) as detected by Digene (61\%), cases with high p634A4 index were still more likely to have subsequent high-grade lesions detected $(P=0.016)$. Among low-grade lesions, significantly higher TAp73 $(P=0.038)$ was found in cases that subsequently progressed to high-grade lesions. There was significant correlation between presence of high-risk HPV and $p 634 A 4$ index $(P=0.01)$. In summary, $p 63$ and p73 immunocytochemistry are potential good markers for detection of carcinoma and high-grade lesions in cervical cytology samples and for triage management of women with atypical squamous cells and low-grade lesions.
\end{abstract}

Modern Pathology (2010) 23, 559-566; doi:10.1038/modpathol.2009.198; published online 15 January 2010

Keywords: $p 63 ; p 73$; liquid-based cervical cytology

Cervical cytology screening is effective in the prevention of cervical cancer by detecting asymptomatic cervical cancer and its precursors although there is limitation in its sensitivity and specificity. ${ }^{1}$ Among cytological abnormalities detectable in a screening population, atypical squamous cells of undetermined significance (ASC-US) and low-grade squamous intraepithelial lesion (LSIL), especially

Correspondence: Professor ANY Cheung, MD, FRCPath, FHKAM(Path), FIAC, Department of Pathology, The University of Hong Kong, Queen Mary Hospital, University Pathology Building, 102 Pokfulam Road, Hong Kong, China.

E-mail: anycheun@hkucc.hku.hk

Received 6 April 2009; revised and accepted 8 May 2009; published online 15 January 2010 the former, are the most common diagnostic categories. $^{2-4}$ Both were associated with a higher possibility of subsequent development of cervical cancer when compared with women with negative cytology. ${ }^{3}$ Colposcopic examination of all such cases poses significant demand on the community resources. To improve the efficiency of cervical cancer screening, human papillomaviruses (HPV) DNA testing has been introduced for the triage of women with ASC-US and for primary screening. ${ }^{5,6}$ Although HPV test shows high sensitivity, additional markers are necessary to improve the specificity of identifying cervical cancers or high-grade precursors. ${ }^{7-11}$ It is also necessary to discover adjunct markers for management of LSIL as HPV testing is not so effective in triage management of LSIL. 
$p 63$ and $p 73$ belong to the family of $p 53$ proteins. $p 53$ is the most extensively studied tumor suppressor gene in human cancers. p63 and p73, share both structural homology and functional similarities with p53. The latter includes activation of p53downstream genes, regulation of cell cycle checkpoints and induction of apoptosis. ${ }^{12-14}$ However, unlike p53, inactivating mutations of p63 and p73 in cancers are uncommon. ${ }^{12-14}$ On the other hand, multiple isoforms of $\mathrm{p} 63^{15-17}$ and $\mathrm{p} 73^{16,18}$ exist.

The full-length wild-type isoforms of $p 63$ (TAp63) and $p 73$ (TAp73) contain an NH2-terminal transactivation domain (TA) that can activate downstream target genes and induce apoptosis. In contrast, the NH2-terminal truncated forms, $\Delta N p 63$ and $\Delta N p 73$, lack the TA and exert negative regulatory effect on p53 and wild-type TAp73 and execute anti-apoptotic function. As a result, TAp63 and TAp73 are p53-synergistic, anti-oncogenic and pro-apoptotic nature, whereas $\Delta N p 73$ are oncogenic and anti-apoptotic ${ }^{15,16,18-21}$

Aberrant expression of p63 isoforms has been found in several human cancers ${ }^{22-26}$ in association with specific cell type differentiation and progress of cancers. Dysregulated expression of $p 73$ variants has been studied even more extensively. These studies, including our previous reports on cervical cancers, suggest that $p 73$ isoforms are involved in human carcinogenesis correlating with prognosis, radiosensitivity and chemosensitivity. ${ }^{27-31}$

In this study, we evaluated the expression of TAp73 and p634A4 isoforms in liquid-based cervical cytology samples of different diagnostic categories and correlated with follow-up profile of such cases.

\section{Materials and methods}

\section{Clinical Samples}

The residues of 529 ThinPrep-processed (Cytyc, Boxborough, MA, USA) cervical cytology samples were collected from the Department of Pathology, the University of Hong Kong, Queen Mary Hospital. The samples include 91 high-grade squamous intraepithelial lesions (HSIL), 107 LSILs, 212 ASCUS, 63 normal samples and 56 invasive cervical squamous cell carcinomas (Table 1). All except 47 carcinomas were archived samples from the Cervical Cytology Laboratory of the University of Hong Kong and they were collected from a screening population in Hong Kong. ${ }^{2}$ The remaining cancer cases were collected from the laboratory and clinics of Queen Mary Hospital. The use of cytology residues was approved by the Institutional Ethics Review Board.

All the cases of ASC-US have been tested for highrisk HPV by the Digene Hybrid Capture Test II Test (Qiagen, USA). The cytology and histology followup data of the patients within 2-year after initial cytology diagnosis were retrieved. Such follow-up
Table 1 Age and cytological diagnosis distribution of women whose samples were included in the study

\begin{tabular}{lccccr}
\hline & \multicolumn{5}{c}{ Number of samples } \\
\cline { 2 - 6 } Age & Negative & ASC-US & LSIL & HSIL & CA \\
\hline$<20$ & 3 & 2 & 4 & 1 & 0 \\
$20-29$ & 14 & 41 & 20 & 12 & 0 \\
$30-39$ & 17 & 80 & 19 & 27 & 6 \\
$40-49$ & 16 & 75 & 52 & 35 & 5 \\
$50-59$ & 12 & 13 & 13 & 11 & 25 \\
$>60$ & 1 & 1 & 4 & 5 & 20 \\
& 63 & 212 & 112 & 91 & 56 \\
\hline
\end{tabular}

CA, squamous cell carcinoma.

data were available in 193/212 (91\%) of ASCUS and $106 / 107(100 \%)$ of LSIL cases.

\section{Cell Lines}

The human cervical squamous cell carcinoma cell lines C4-1 and ME180 were obtained from American Type Culture Collection (Manassas, VA, USA) and cultured in MEM (Life Technologies Inc., Gaithersburg, MD, USA) supplemented with $10 \%$ fetal bovine serum and $1 \%$ penicillin and streptomycin. ${ }^{30}$

\section{Preparation of Slides}

The cell pellet of the liquid-based cytology residues of the above clinical samples collected after centrifuge was resuspended in PreservCyt solution. C41 and ME180 cells were detached from culture flasks, washed with PBS and fixed in PreservCyt solution. Thin-layer cytology slides were made using the ThinPrep 2000 Processor and were fixed in $95 \%$ alcohol $^{9}$ until use for immunocytochemistry.

\section{Immunocytochemistry}

Thin-layer cytology slides were dried overnight in an oven at $37^{\circ} \mathrm{C}$. Antigen retrieval was performed by heating immersed slides in $10 \mathrm{mM}$ sodium citrate buffer, $\mathrm{pH} 6.0$, at $95^{\circ} \mathrm{C}$ in a microwave oven for $15 \mathrm{~min}$. Endogenous peroxidases were blocked by treatment with $3 \% \mathrm{H}_{2} \mathrm{O}_{2}$ in methanol. Mouse antihuman p63, Clone 4A4 (Dako, Glostrup, Denmark) and mouse anti-human TAp73 (Zymed laboratories, CA, USA) were applied at the dilution of 1:1000 and 1:100, respectively. The anti-p63 $4 A 4$ antibody reacts with both TAp63 and $\triangle N p 63$ isoforms, whereas the anti$T A p 73$ antibody detects the TAp73 $\alpha$ isoform. Immunocytochemistry was performed using EnVision + Dual Link System (Dako, Carpinteria, CA, USA). ${ }^{32}$ A light hematoxylin counterstain was used. The cervical carcinoma cell lines ME $180^{33}$ and C4-1 were used as positive control for $p 63$ and $p 73$, respectively. 
Table 2 Scoring system for the assessment of p634A4 and TAp73 immunoreactivity (modified from Wentzensen, $\mathrm{N}$ et al)

\begin{tabular}{lll}
\hline Score & Staining pattern & Interpretation \\
\hline 0 & $\begin{array}{l}\text { p634A4/TAp73 negative } \\
\text { p634A4/TAp73 positive but not in }\end{array}$ & $\begin{array}{l}\text { Negative } \\
\text { atypical cells }\end{array}$ \\
$\begin{array}{l}\text { p634A4/TAp73 positive in atypical cells } \\
\text { but scanty }\end{array}$ & Positive \\
& $\begin{array}{l}\text { p634A4/TAp73 positive in moderate } \\
\text { numbers of atypical cell }\end{array}$ & Positive \\
& $\begin{array}{l}\text { p634A4/TAp73 positive in most atypical } \\
\text { cells }\end{array}$ & Positive \\
& & \\
\hline
\end{tabular}

Replacement of the primary antibody with preimmune IgG serum was used as a negative control. The experimental conditions were uniform for all samples.

\section{Scoring for $p 63$ and $p 73$ Immunoreactivity}

A modified Wentzensen scoring system ${ }^{34}$ incorporating morphologic criteria was used for the assessment of the immunoreactivity of the cells for TAp73 and p634A4 (Table 2). The whole slide was screened in each case. More than 10 epithelial groups in each slide with the highest immunoreactivity were assessed. Cells with atypical cytological features were evaluated for immunoreactivity and an immunocytochemical index of $0-4$ was obtained. The immunoreactivity for TAp73 (Tsun and Cheung) and p634A4 (Ng and Cheung) in the samples were evaluated by two authors. If indicated, cases with discrepancy in scoring were discussed under multihead microscope. The specific cytological diagnosis of the samples was disclosed after the scoring was performed.

\section{Statistical Analysis}

Statistical analysis was performed using the Statistical Package for Social Science 16 for Windows (SPSS Inc., Chicago, IL, USA). The significance of difference in TAp73 and p634A4 expression among all cervical cytology diagnostic categories was examined by non-parametric Kruskal-Wallis $H$ rank test and Chi-square test. The significance of difference between any two diagnostic groups was analyzed by non-parametric Mann-Whitney $U$ test. Correlation coefficient was calculated by Pearson correlation test. All statistical tests were calculated in two-sided and $P$-value of $<0.05$ was considered statistically significant.

\section{Results}

The immunoreactivity for both p634A4 and TAp73 was predominantly found at the nuclei though weak cytoplasmic expression was focally and occasion- ally found (Figure 1). There was no apparent effect of storage on the immunoreactivity. A comparison of the p634A4 and TAp73 indices in different categories of cervical cytology diagnosis was summarized in Figure 2 and Table 3. Although significant correlation between TAp73 and p634A4 indices $(P<0.0001$, Pearson test $)$ was found, statistically significant difference in both indices among the different diagnostic categories $(P<0.0001$, Kruskal-Wallis test) was observed.

When compared with negative smears, ASC-US showed significant higher TAp73 index $(P<0.0001$, Mann-Whitney test) although no significant difference in p634A4 index was noted between the two categories. There was also no significant difference in TAp73 and p634A4 indices between ASC-US and LSIL. HSIL, however, showed significant higher TAp73 and p634A4 indices than LSIL $(P<0.0001$, $P<0.0001$, Mann-Whitney test). The two antibodies could also detect isolated small HSIL cells. There was no significant difference in TAp73 and p634A4 indices between HSIL and carcinomas.

We were able to retrieve the follow-up cytology and/or histology data in 193/212 (91\%) of ASC-US and $106 / 107(99.6 \%)$ of LSIL cases. Correlation between TAp73 and p634A4 indices in ASC-US samples and subsequent development of LSIL/HSIL were shown in Table 4. Among cases of ASC-US, significantly higher p634A4 index was found in cases that subsequently progressed to LSIL $(P=$ 0.031) or HSIL $(P=0.006)$ or above than those that did not. On the other hand, there was no statistically significant correlation with TAp73 index and subsequently development of LSIL $(P=0.952)$ or HSIL $(P=0.365)$.

Among the 212 ASC-US, 129 (61\%) were positive for high-risk HPV as detected by Digene test. There was significant correlation between presence of high-risk HPV and $p 634 A 4$ index $(P=0.01)$ but not the TAp73 index $(P=0.891)$. Among ASC-US cases positive for HPV, cases with high p634A4 index were more likely to have subsequent HSIL detected $(P=0.016)$ whereas no predictive significance was found for subsequent detection of LSIL $(P=0.124)$. There was no significant correlation in TAp73 index between HPV-positive ASC-US cases that have LSIL $(P=1)$ or HSIL $(P=0.42)$ or above subsequently detected.

Among LSIL, significantly higher TAp73 index $(P=0.038)$ was also found in those cases that subsequently progressed to HSIL or above than those that did not (negative, ASC-US or LSIL) but no significant difference was observed for p634A4 $(P=0.689)$.

\section{Discussion}

\section{Novel Markers for Cervical Cytology}

Cervical cancer screening by cytomorphological evaluation is one of the most successful cancer 

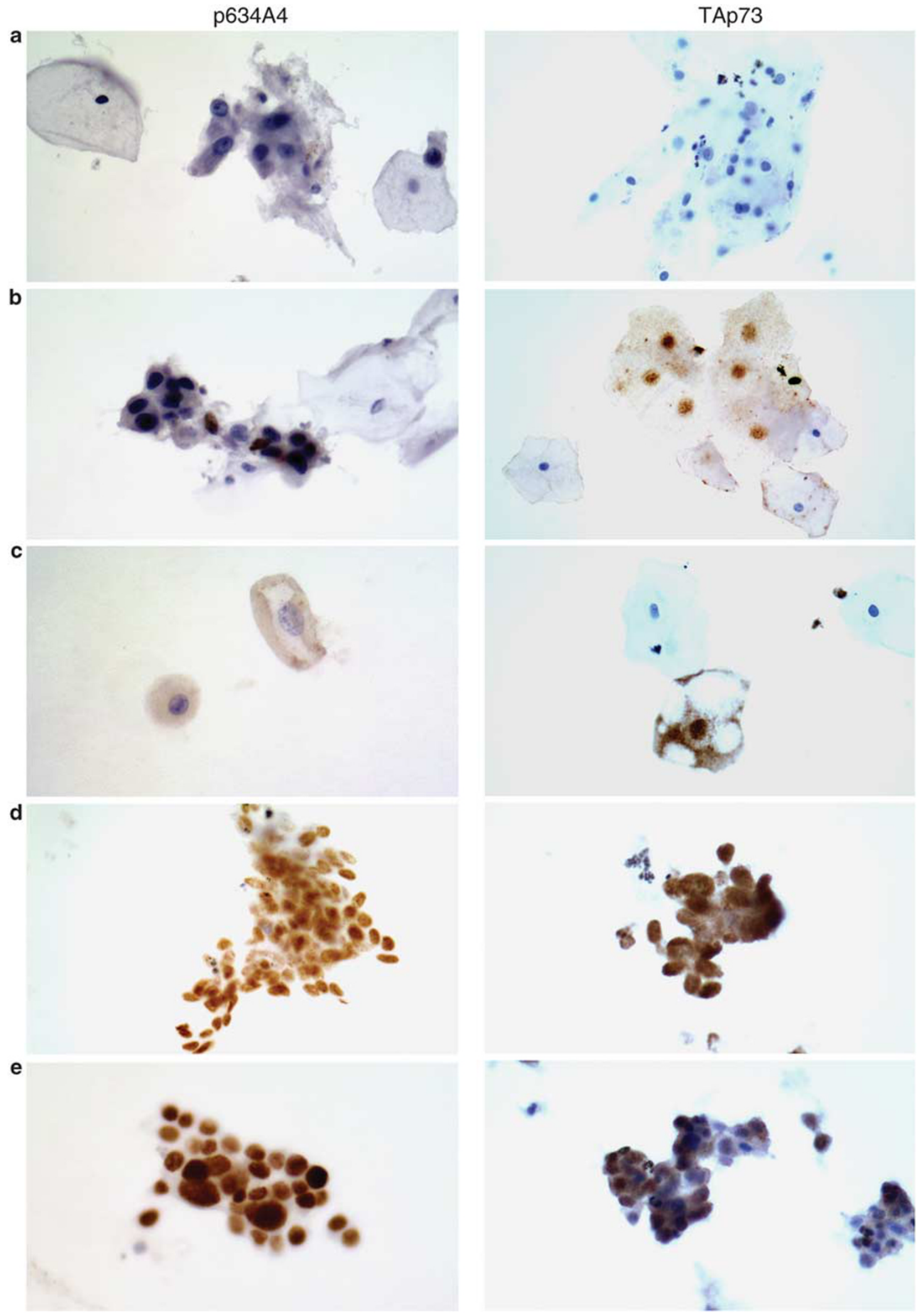

Figure 1 p634A4 and TAp73 expression was assessed in liquid-based cytology by immunocytochemistry (magnification: 10 $\times 40$ ): (a) Normal squamous cells are negative for both p634A4 and TAp73. Focal weak to moderate immunoreactivity of p634A4 and TAp73 was noted in ASC-US (b) and LSIL (c). HSIL (d) and squamous cell carcinoma (e) cells were strongly positive for both p634A4 and TAp73. 

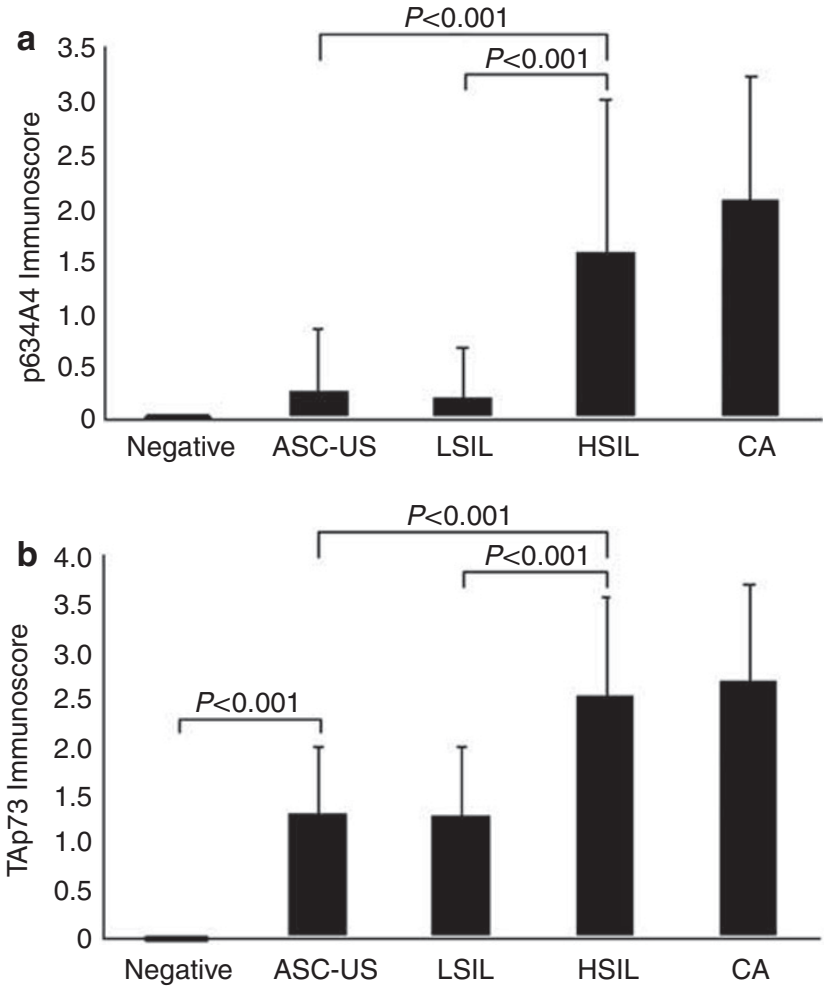

Figure 2 p634A4 (a) and TAp73 (b) protein expression in normal cervical cells, ASC-US, LSIL, HSIL and squamous cell carcinoma (CA) were analyzed by Mann-Whitney test.

Table 3 Comparison of TAp73 and p634A4 indices comparing different categories of cervical cytology diagnosis

\begin{tabular}{lcc}
\hline & $\begin{array}{c}\text { P-value (TAp73 } \\
\text { index) }\end{array}$ & $\begin{array}{c}\text { P-value (p634A4 } \\
\text { index) }\end{array}$ \\
\hline $\begin{array}{l}\text { Negative vs ASC- } \\
\text { US }\end{array}$ & $\leq 0.001$ & 0.524 \\
ASC-US vs LSIL & 0.606 & 0.540 \\
ASC-US vs HSIL & $\frac{<0.001}{<0.001}$ & $\frac{<0.001}{<0.001}$ \\
LSIL vs HSIL & $\frac{0.355}{0.084}$ \\
HSIL vs CA & & $\frac{0.04}{}$ \\
\hline
\end{tabular}

CA, squamous cell carcinoma.

$P$-values were calculated by Mann-Whitney test. Those with significant $P$-values are underlined.

screening programs. Nevertheless, there still exist limitations in cervical cytology screening. New laboratory techniques are therefore being continuously explored to improve sensitivity and specificity of cervical cytology evaluation. The liquid-based cytologic preparation was popularly applied in the recent decade. It allows collection of exfoliated cervical epithelial cells in liquid buffer, reduces the interferring background of blood and inflammatory cells and improves the quality of the sample for screening of cancer cells. ${ }^{2}$ Moreover, after the production of a thin layered cervical smear, the residual cells in the vial can conveniently be used for ancillary investigations.
Table 4 Correlation between TAp73 and p634A4 indices in ASCUS samples and subsequent development of LSIL/HSIL or above

$\begin{array}{cc}\text { P-value } & \text { P-value } \\ \text { (TAp73 } & \text { (p634A4 } \\ \text { index) } & \text { index) }\end{array}$

Among ASC-US

Subsequent development of LSIL+

Subsequent development of HSIL+

0.952

0.365

$\underline{0.031}$

Among HPV-positive ASC-US

Subsequent development of LSIL+ $\quad 1 \quad 0.124$

Subsequent development of HSIL+ $\quad 0.42 \quad \underline{0.016}$

Among LSIL

Subsequent development of HSIL+ $\quad \underline{0.038} \quad 0.689$

LSIL+, LSIL or above; HSIL+, HSIL or above.

$P$-values were calculated by Mann-Whitney test. Those with significant P-values are underlined.

HPV molecular test performed on directly collected samples or from liquid-based cell residues is currently the most commonly applied ancillary test to cervical cytology.,6,10 It enables triage of management of women with ASC-US, primary screening and followup of patients with cervical neoplasia. Besides HPV tests, cervical cytology samples can also be used for other evaluation including genetic and epigentic studies. ${ }^{6,10}$ The ploidy of epithelial cells and the immunohistochemical detection of expression of genetic markers can be assessed with an intact morphological background by immunocytochemistry and in situ hybridization enhancing identification of dysplastic cells. ${ }^{8,9,35,36}$ For instance, p16 immunohistochemical study on liquid-based cytology has been reported to be particularly hopeful in detection of cancer cells and their precursors although there still exist limitations in its application. ${ }^{6,36}$ Further exploration of novel biomarkers for identifying cervical cancers and their precursors is still an ongoing process.

We believe that combined cytopathologic evaluation and application of molecular markers in cervical cytology is a promising option to facilitate reliable detection of cervical cancer. Owing to the important roles of $p 63$ and $p 73$, the two homologues of $p 53$, in cervical epithelial differentiation and carcinogenesis, the significance of immunoreactivity for $p 634 A 4$ and TAp73 in liquid-based cervical cytology was evaluated in this study. The antip634A4 antibody recognizes both $T A p 63$ and $\Delta N p 63$ isoforms, whereas the anti-TAp73 antibody detects the TAp73 $\alpha$ isoform. They were selected for the study based on our previous experience on these two genes. ${ }^{25,26,29,37}$

\section{P63 and Cervical Epithelial Differentiation and Carcinogenesis}

Upregulated expression of both TAp63 and $\Delta N p 63$ isoforms has been demonstrated in cervical intra- 
epithelial neoplasia and invasive cancers. ${ }^{4,33}$ The expression of $p 63$ gene was found to be associated with poor survival and local recurrence of cervical squamous cell carcinoma. ${ }^{38}$ In this study, p634A4 immunoreactivity was found to be significantly higher in HSIL and cancers. The antibody can also detect isolated small HSIL cells and can improve the sensitivity of detecting these 'litigation' cells that are potentially easy to be missed.

P63 has been suggested to be useful to distinguish squamous and glandular lesions in cervical samples $^{39}$ and the endocervical glandular cells in this study was found to be negative for p634A4. In the squamous mucosa, p63 expression is often localized to the basal and parabasal cells of mature or metaplastic stratified squamous epithelium but may be present in all cell layers in atrophic epithelium. p63 is indeed considered as a marker for reserve cells or stem cell like cells. ${ }^{25,40-42}$ p63 expression by immunostaining alone may therefore highlight basal cells, which may become a diagnostic pitfall in atrophic samples. By combining p63 immunoreactivity assessment and morphological evaluation, such false positive results may be minimized.

\section{P73 and Cervical Cancers}

Dysregulated expression of $p 73$ isoforms in cervical intraepithelial neoplasia and cervical cancer has been reported. ${ }^{29,43,44}$ In our earlier studies, the expressions of $\triangle N p 73$ and $T A p 73$ were found to be independently associated with prognosis of patients with cervical cancers. The two p73 isoforms have been found to be potential markers for predicting the prognosis and sensitivity to radiotherapy in patients with cervical squamous cell carcinoma. ${ }^{29}$ In this study, TAp73 immunoreactivity was also found to be higher in HSIL and squamous cell carcinomas.

\section{Correlation with Progress of Disease}

CIN is actually a dynamic lesion capable of spontaneous regression or progression into more serious lesion including cancers. It is believed that biomarkers such as p16 can help in evaluating the likelihood for progression and regression in a particular CIN. ${ }^{7}$

Such markers may be particularly useful in assisting triage of patients with borderline smears reported as ASC-US. Our study on an Asian screening population in Hong Kong showed that, similar to other screening populations, ASC-US is the most common abnormal cytologic finding ${ }^{2}$ and is associated with a significantly higher chance to develop cervical cancer and its high-grade precursors. ${ }^{3} \mathrm{HPV}$ DNA test has been used for triage of ASCUS. However, majority of the ASC-US positive for highrisk HPV does not progress to high-grade cervical intraepithelial lesion. Management problem in women with LSIL also exists. Although more than $50 \%$ of LSIL will regress, HSIL or above can still be found in a significant portion of these women. ${ }^{45}$ Women with LSIL may be referred for colposcopy or followed up with cytology with variation in management guidelines in different countries.

In this study, high p634A4 immunoreactivity correlated with higher likelihood of progressing to LSIL or HSIL lesions in subsequent cytology or histology follow-up among ASC-US cases independent of their HPV status. On the other hand, high TAp73 index in LSIL correlated with higher chance of progressing to HSIL or above. p634A4 may thus be a promising marker to further triage management of women with HPV-positive ASC-US whereas TAp73 is a good marker for deciding management of women with LSIL. Ancillary immunocytochemical studies for p634A4 and TAp73 can be performed in ASC-US and LSIL samples, respectively. Those with high p634A4 and TAp73 expression will be referred for colposcopy whereas those with low expression can be followed up with repeat cytology. This will reduce the burden of unnecessary colposcopy.

\section{Reaction with HPV}

HPV are recognized carcinogen for cervical cancers and their precursors. The E6 and E7 genes of highrisk HPV genotypes are essential in promoting degradation and/or inactivation of the p53 and retinoblastoma proteins, respectively, resulting in disruption of the important tumor suppressor functions of these two genes.

It is controversial whether there is a direct interaction between $p 63$ and $p 73$ with HPV viral oncoproteins. Interaction among HPV, $p 63$ and $p 73$ has been reported. A strong circumstantial association between p63 immunoreactivity and HPV 16 has been reported in earlier study because of their colocalization in squamous cell carcinomas. ${ }^{46}$ Indeed, p63 is considered as a HPV target cell. ${ }^{41}$ Recently, Teisser et $a l^{47}$ reported that the $p 63$ target genes could be activated by suppressing the E6/ E6AP pathway. In our study, significant correlation between presence of high-risk HPV and p634A4 index was found among cases of ASC-US.

Park et $a l^{48}$ showed that $p 73$ could also be inactivated by HPV-E6, but not by ubiquitin/proteasome-dependent mechanisms as in the case of $p 53$. p73 promoter has also been shown to be affected, at least in cell lines and primary human keratinocytes, by E7 proteins from oncogenic HPV types. ${ }^{43}$ Studies on $p 73-/-$ HPV38 E6/E7 transgenic mice keratinocytes also showed that loss of $p 53$ could stop $\Delta N p 73$ expression and partially restore the UV-activated cell cycle checkpoints. On the other hand, loss of $\Delta N p 73$ led to restoration of the p53 pathways. ${ }^{49} \mathrm{In}$ this study, however, the $\Delta N p 73$ immunohistochemistry did not correlate with the HPV status. 
In summary, in this immunocytochemical study on a relatively large number of liquid-based cytology samples, we showed that TAp73 and p634A4 immunoreactivity together with morphological assessment could distinguish cervical cancer and the high-grade precursors. In particular, p634A4 and TAp73 immunoreactivity correlated with subsequent detection of HSIL or above in patients with ASC-US and LSIL, respectively. Cases of ASC-US positive for p634A4 are also more likely to harbor high-risk HPV. p634A4 and TAp73 immunoreactivity may thus be useful potential markers for triage of borderline and low-grade cervical smears, respectively.

\section{Acknowledgements}

This study was supported by the Research Fund for the Control of Infectious Diseases/Health and Health Services Fund (Project Number: 06060642). The high-risk HPV Hybrid Capture Test II was supported by the SK Yee Medical Foundation.

\section{Disclosure/conflict of interest}

The authors declare no conflict of interest.

\section{References}

1 Peto J, Gilham C, Fletcher O, et al. The cervical cancer epidemic that screening has prevented in the UK. Lancet 2004;364:249-256.

2 Cheung AN, Szeto EF, Leung BS, et al. Liquid-based cytology and conventional cervical smears: a comparison study in an Asian screening population. Cancer 2003;99:331-335.

3 Cheung AN, Szeto EF, Ng KM, et al. Atypical squamous cells of undetermined significance on cervical smears: follow-up study of an Asian screening population. Cancer 2004;102:74-80.

4 Quade BJ, Yang A, Wang Y, et al. Expression of the p53 homologue p63 in early cervical neoplasia. Gynecol Oncol 2001;80:24-29.

5 Castle PE, Sadorra M, Garcia F, et al. Pilot study of a commercialized human papillomavirus (HPV) genotyping assay: comparison of HPV risk group to cytology and histology. J Clin Microbiol 2006;44:3915-3917.

6 Cuzick J, Arbyn M, Sankaranarayanan R, et al. Overview of human papillomavirus-based and other novel options for cervical cancer screening in developed and developing countries. Vaccine 2008;26(Suppl 10): K29-K41.

7 Baak JP, Kruse AJ, Robboy SJ, et al. Dynamic behavioural interpretation of cervical intraepithelial neoplasia with molecular biomarkers. J Clin Pathol 2006; 59:1017-1028.

8 Ngan HY, Cheung AN, Liu SS, et al. Telomerase assay and HPV 16/18 typing as adjunct to conventional cytological cervical cancer screening. Tumour Biol 2002;23:87-92.
9 Cheung AN, Chiu PM, Tsun KL, et al. Chromosome in situ hybridisation, Ki-67, and telomerase immunocytochemistry in liquid based cervical cytology. J Clin Pathol 2004;57:721-727.

10 Cheung AN. Molecular targets in gynaecological cancers. Pathology 2007;39:26-45.

11 Cuschieri K, Wentzensen N. Human papillomavirus mRNA and p16 detection as biomarkers for the improved diagnosis of cervical neoplasia. Cancer Epidemiol Biomarkers Prev 2008;17:2536-2545.

12 Nishi H, Isaka K, Sagawa Y, et al. Mutation and transcription analyses of the p63 gene in cervical carcinoma. Int J Oncol 1999;15:1149-1153.

13 Yang A, McKeon F. P63 and P73: P53 mimics, menaces and more. Nat Rev Mol Cell Biol 2000;1:199-207.

14 Irwin MS, Kaelin WG. p53 family update: p73 and p63 develop their own identities. Cell Growth Differ 2001;12:337-349.

15 Yang A, Kaghad M, Wang Y, et al. p63, a p53 homolog at 3q27-29, encodes multiple products with transactivating, death-inducing, and dominant-negative activities. Mol Cell 1998;2:305-316.

16 Moll UM, Slade N. p63 and p73: roles in development and tumor formation. Mol Cancer Res 2004;2:371-386.

17 Finlan LE, Hupp TR. p63: the phantom of the tumor suppressor. Cell Cycle 2007;6:1062-1071.

18 Melino G, De Laurenzi V, Vousden KH. p73: friend or foe in tumorigenesis. Nat Rev Cancer 2002;2:605-615.

19 Jost CA, Marin MC, Kaelin Jr WG. p73 is a simian (correction of human) p53-related protein that can induce apoptosis. Nature 1997;389:191-194.

20 Zhu J, Jiang J, Zhou W, et al. The potential tumor suppressor p73 differentially regulates cellular p53 target genes. Cancer Res 1998;58:5061-5065.

21 Grob TJ, Novak U, Maisse C, et al. Human delta Np73 regulates a dominant negative feedback loop for TAp73 and p53. Cell Death Differ 2001;8:1213-1223.

22 Urist MJ, Di Como CJ, Lu ML, et al. Loss of p63 expression is associated with tumor progression in bladder cancer. Am J Pathol 2002;161:1199-1206.

23 Stefansson IM, Salvesen HB, Akslen LA. Loss of p63 and cytokeratin 5/6 expression is associated with more aggressive tumors in endometrial carcinoma patients. Int J Cancer 2006;118:1227-1233.

24 Narahashi T, Niki T, Wang T, et al. Cytoplasmic localization of p63 is associated with poor patient survival in lung adenocarcinoma. Histopathology 2006;49:349-357.

25 Liao XY, Xue WC, Shen DH, et al. p63 expression in ovarian tumours: a marker for Brenner tumours but not transitional cell carcinomas. Histopathology 2007;51: 477-483.

26 Zhang HJ, Xue WC, Siu MK, et al. P63 expression in gestational trophoblastic disease: correlation with proliferation and apoptotic dynamics. Int J Gynecol Pathol 2009;Jan:30.

27 Coates PJ. Regulating p73 isoforms in human tumours. J Pathol 2006;210:385-389.

28 Dominguez G, Garcia JM, Pena C, et al. DeltaTAp73 upregulation correlates with poor prognosis in human tumors: putative in vivo network involving p73 isoforms, p53, and E2F-1. J Clin Oncol 2006;24:805-815.

29 Liu SS, Leung RC, Chan KY, et al. p73 expression is associated with the cellular radiosensitivity in cervical cancer after radiotherapy. Clin Cancer Res 2004;10: 3309-3316.

30 Liu SS, Chan KY, Leung RC, et al. Enhancement of the radiosensitivity of cervical cancer cells by over- 
expressing p73alpha. Mol Cancer Ther 2006;5: 1209-1215.

31 Concin N, Hofstetter G, Berger A, et al. Clinical relevance of dominant-negative p73 isoforms for responsiveness to chemotherapy and survival in ovarian cancer: evidence for a crucial p53-p73 cross-talk in vivo. Clin Cancer Res 2005;11:8372-8383.

32 Siu MK, Wong ES, Chan HY, et al. Overexpression of NANOG in gestational trophoblastic diseases: effect on apoptosis, cell invasion, and clinical outcome. Am J Pathol 2008;173:1165-1172.

33 Lin Z, Nan Y, Zhang X, et al. Reverse transcriptionpolymerase chain reaction and western blotting analysis for detection of p63 isoforms in uterine cervical cancers. Int J Gynecol Cancer 2006;16:1643-1647.

34 Wentzensen N, Bergeron C, Cas F, et al. Evaluation of a nuclear score for p16INK4a-stained cervical squamous cells in liquid-based cytology samples. Cancer 2005; 105:461-467.

35 Sahebali S, Depuydt CE, Boulet GA, et al. Immunocytochemistry in liquid-based cervical cytology: analysis of clinical use following a cross-sectional study. Int J Cancer 2006;118:1254-1260.

36 Yoshida T, Fukuda T, Sano T, et al. Usefulness of liquidbased cytology specimens for the immunocytochemical study of p16 expression and human papillomavirus testing: a comparative study using simultaneously sampled histology materials. Cancer 2004;102:100-108.

37 Liu SS, Chan KY, Cheung AN, et al. Expression of deltaNp73 and TAp73alpha independently associated with radiosensitivities and prognoses in cervical squamous cell carcinoma. Clin Cancer Res 2006;12: 3922-3927.

38 Cho NH, Kim YB, Park TK, et al. P63 and EGFR as prognostic predictors in stage IIB radiation-treated cervical squamous cell carcinoma. Gynecol Oncol 2003;91:346-353.

39 Garcia MT, Acar BC, Jorda M, et al. Use of p63 for distinction of glandular versus squamous lesions in cervicovaginal specimens. Cancer 2007;111:54-57.
40 Di Como CJ, Urist MJ, Babayan I, et al. p63 expression profiles in human normal and tumor tissues. Clin Cancer Res 2002;8:494-501.

41 Martens JE, Arends J, Van der Linden PJ, et al. Cytokeratin 17 and p63 are markers of the HPV target cell, the cervical stem cell. Anticancer Res 2004;24: 771-775.

42 Witkiewicz AK, Hecht JL, Cviko A, et al. Microglandular hyperplasia: a model for the de novo emergence and evolution of endocervical reserve cells. Hum Pathol 2005;36:154-161.

43 Brooks LA, Sullivan A, O’Nions J, et al. E7 proteins from oncogenic human papillomavirus types transactivate p73: role in cervical intraepithelial neoplasia. Br J Cancer 2002;86:263-268.

44 Nenutil R, Ceskova P, Coates PJ, et al. Differential expression of p73alpha in normal ectocervical epithelium, cervical intraepithelial neoplasia, and invasive squamous cell carcinoma. Int J Gynecol Pathol 2003; 22:386-392.

45 Wright Jr TC, Cox JT, Massad LS, et al. 2001 Consensus Guidelines for the management of women with cervical cytological abnormalities. JAMA 2002;287: 2120-2129.

46 Wang TY, Chen BF, Yang YC, et al. Histologic and immunophenotypic classification of cervical carcinomas by expression of the p53 homologue p63: a study of 250 cases. Hum Pathol 2001;32:479-486.

47 Teissier S, Ben Khalifa Y, Mori M, et al. A new E6/P63 pathway, together with a strong E7/E2F mitotic pathway, modulates the transcriptome in cervical cancer cells. J Virol 2007;81:9368-9376.

48 Park JS, Kim EJ, Lee JY, et al. Functional inactivation of p73, a homolog of p53 tumor suppressor protein, by human papillomavirus E6 proteins. Int J Cancer 2001;91:822-827.

49 Dong W, Arpin C, Accardi R, et al. Loss of p53 or p73 in human papillomavirus type $38 \mathrm{E} 6$ and E7 transgenic mice partially restores the UV-activated cell cycle checkpoints. Oncogene 2008;27:2923-2928. 